\title{
Spatial inattention abolishes voice adaptation
}

\author{
Romi Zäske • Christiane Fritz • Stefan R. Schweinberger
}

Published online: 12 January 2013

(C) Psychonomic Society, Inc. 2013

\begin{abstract}
Adaptation to male voices causes a subsequent voice to be perceived as more female, and vice versa. Similar contrastive aftereffects have been reported for phonetic perception, and in vision for face perception. However, while aftereffects in the perception of phonetic features of speech have been reported to persist even when adaptors were processed inattentively, face aftereffects were previously reported to be abolished by inattention to adaptors. Here we demonstrate that auditory aftereffects of adaptation to voice gender are eliminated when the male and female adaptor voices are spatially unattended. Participants simultaneously heard genderspecific male or female adaptor voices in one ear and gender-neutral (androgynous) adaptor voices in the contralateral ear. They selectively attended to the adaptor voices in a designated ear, by either classifying voice gender (Exp. 1) or spoken syllable (Exp. 2). Voice aftereffects were found only if the gender-specific voices were spatially attended, suggesting capacity limits in the processing of voice gender for the unattended ear. Remarkably, gender-specific adaptors in the attended ear elicited comparable aftereffects in test voices, regardless of prior attention to voice gender or phonetic content. Thus, within the attended ear, voice gender was processed even when it was irrelevant for the task at hand, suggesting automatic processing of gender along with
\end{abstract}

\footnotetext{
R. Zäske $(\triangle) \cdot$ C. Fritz $\cdot$ S. R. Schweinberger

Department for General Psychology and Cognitive Neuroscience, Institute of Psychology, Friedrich Schiller University Jena, Am Steiger 3, Haus 1,

D-07743 Jena, Germany

e-mail: romi.zaeske@uni-jena.de

R. Zäske · S. R. Schweinberger

DFG Research Unit Person Perception, Institute of Psychology,

Friedrich Schiller University of Jena, Am Steiger 3, Haus 1,

D-07743 Jena, Germany
}

linguistic information. Overall, voice gender adaptation requires spatial, but not dimensional, selective attention.

Keywords Auditory aftereffects · Adaptation · Voice gender $\cdot$ Selective attention

Recent experience can dramatically alter the way that subsequent stimuli are perceived. For instance, prolonged exposure to unidirectional visual or auditory motion causes transient aftereffects, such that subsequent stationary objects are perceived as moving in the opposite direction (Anstis, Verstraten, \& Mather, 1998; Grantham \& Wightman, 1979). Contrastive aftereffects of adaptation are believed to reveal neuronal coding of specific stimulus properties and are well-known for the perception of various low-level features, such as motion or color. In the past decade, using sophisticated digital "morphing" software, a striking finding has been that adaptation also influences human perception of complex social signals. For instance, adaptation to male faces causes androgynous faces to be perceived as more female, and vice versa, and similar contrastive aftereffects were also seen for the perception of facial expressions, ethnicity, or identity (Leopold, O’Toole, Vetter, \& Blanz, 2001; Webster, Kaping, Mizokami, \& Duhamel, 2004).

In the 1970s, adaptation paradigms have also been extensively employed in speech perception research to study the representation of various linguistic features such as voicing (Eimas \& Corbit, 1973), place of articulation (Cooper \& Blumstein, 1974), or vowel quality (Morse, Kass, \& Turkienicz, 1976). For instance, in their seminal study, Eimas and Corbit demonstrated that adaptation to [ba] or [pa] syllables contrastively biases the perception of subsequent test stimuli that randomly vary in voice onset time (VOT) between [ba] and [pa]. Specifically, test stimuli were more likely to be perceived as [pa] after [ba] adaptation, and 
vice versa. This shift of the category boundary toward the adapting stimulus was interpreted in terms of selective fatigue of phonetic feature detectors sensitive to the respective endpoints of the VOT continuum between prototypical [ba] and [pa]. Other studies further explored the mechanisms underlying contrastive aftereffects in terms of fatigue of feature detectors or changes in the adaptation-level criterion, and tried to disentangle acoustic from phonetic loci of speech aftereffects (e.g., Diehl, Kluender, \& Parker, 1985; Sawusch, 1977; Sawusch \& Jusczyk, 1981; StuddertKennedy, 1980; Tartter \& Eimas, 1975).

Unlike research on linguistic properties of speech, the present study is concerned with adaptation to nonlinguistic information in voices. For instance, auditory aftereffects in the perception of voice gender have been established as being analogous to face gender aftereffects. Specifically, listening to female voices causes subsequent gender-morphed voices to be perceived as more male, and vice versa for listening to male voices (Mullennix, Johnson, Topcu-Durgun, \& Farnsworth, 1995; Schweinberger et al., 2008). In our own research, both low-level adaptation to female or male fundamental frequencies $(f 0)$ and postperceptual adaptation to gender concepts could be excluded as alternative explanations for the voice gender aftereffect, as demonstrated by a series of control experiments (Schweinberger et al., 2008). We therefore reasoned that the voice gender aftereffect acts on a high perceptual level and may reflect adaptation-induced recalibration in the responsivity of neurons that are tuned to male or to female voice quality. The inefficiency of low-level adaptation to sinusoidal tones matched to female or male $f 0$ s is remarkable, both in view of findings that such simple auditory stimuli can alter the perceived gender of androgynous faces (Smith, Grabowecky, \& Suzuki, 2007) and in view of the prominent role of $f 0$ in voice gender perception (Hillenbrand \& Clark, 2009). Neurophysiological evidence for voice gender adaptation has come from a follow-up study showing systematic repetition-induced attenuations in auditory event-related potentials (N1, P2) after adaptation to female or male voices, irrespective of the speech content or speaker identity (Zäske, Schweinberger, Kaufmann, \& Kawahara, 2009). Extending these initial studies, several recent reports have shown that voice adaptation causes transient distortions in the perception of other social signals as well, including voice identity (Latinus \& Belin, 2011; Zäske, Schweinberger, \& Kawahara, 2010), affective information (Bestelmeyer, Rouger, DeBruine, \& Belin, 2010), and age (Zäske \& Schweinberger, 2011). More direct evidence for neuronal adaptation to voice quality has come from electrophysiological and neuroimaging studies, which have suggested decreased neuronal responses to voice repetitions (Andics et al., 2010; Belin \& Zatorre, 2003; Schweinberger, Walther, Zäske, \& Kovacs, 2011) and pointed to a network of brain areas involved in voice gender perception (Charest, Pernet, Latinus, Crabbe, \& Belin, in press).
Several strands of research have suggested remarkably efficient processing of voice signals when they are not directly attended: First, research on the "cocktail-party problem" used dichotic listening to show that changes of speaker gender in one ear are explicitly detected, despite selective spatial attention to messages in the contralateral ear (Cherry, 1953). Moreover, during shadowing of messages presented to one ear, Treisman (1964) reported intrusions of simultaneous messages from the unattended ear as a function of voice similarity. Specifically, intrusions were more likely when messages in the unattended ear were spoken by the same female speaker as those in the attended message (as compared to when messages in the unattended ear were spoken by a male speaker). Treisman concluded that "the difference in voice allows the irrelevant message to be rejected much more efficiently, and this probably takes place at an earlier stage in the perceptual analysis of inputs" (Treisman, 1964, p. 215).

More recently, neuronal processing of complex auditory stimuli has been demonstrated in the absence of directed attention (Näätänen, Paavilainen, Rinne, \& Alho, 2007). Of particular relevance, voice information was found to be processed preattentively, as indexed by the mismatch negativity (MMN), an early $(\sim 150-250 \mathrm{~ms})$ event-related potential that reflects the preattentive detection of auditory changes within a stream of regular input (Näätänen et al., 2007). In an oddball task, Titova and Näätänen (2001) showed that within a stream of a frequent female voice ("standard"), rare voices ("deviants") of other female speakers or of a male speaker elicited an MMN. MMN amplitude was positively correlated with both acoustic and perceived dissimilarity between standard and deviant voices. Beauchemin et al. (2006) conducted an MMN experiment that more clearly separated acoustic and perceptual processes. In their study, a rare familiar voice deviant elicited a greater MMN than did a rare unfamiliar voice deviant within a stream of an unfamiliar standard voice. As this effect was only found for listeners who were personally familiar with deviant voices, this MMN effect must have been driven by higher-level voice representations.

Aftereffects of adaptation have traditionally been conceived of as being purely stimulus-driven - that is, as being independent of directed attention. In line with this notion, speech adaptation studies have suggested that aftereffects on linguistic continua are entirely independent of focused attention to adaptor stimuli (Mullennix, 1986; Samuel \& Kat, 1998; Sussman, 1993). In a complex-dichotic-adaptation paradigm, Mullennix (1986) presented synthetic adaptor syllables ([bæ]) to one ear, embedded within distractor and target syllables. The distractors and targets were naturally produced consonant-vowel (CV) syllables spoken by a man. Simultaneously, the contralateral ear received distractor syllables spoken by a woman. In the "monaural adaptation condition," participants attended to the 
ear containing the synthetic adaptors and the male voice, and labeled the initial consonants of two CV syllables (targets) spoken by the male speaker. The participants pressed a button for one consonant ("primary target") and uttered "stop" when they detected the other consonant ("secondary target"). In order to manipulate the focus of spatial attention toward or away from the synthetic [bæ] adaptors, a "dichotic adaptation condition" was introduced. In that condition, the channel without adaptors - that is, the channel with the female voice - was monitored for primary targets, while at the same time both channels were monitored for secondary targets. In both the monaural and dichotic adaptation conditions, the adaptation phase was followed by test syllables varying on a [bæ]-[dæ] continuum. The test syllables were presented either to the same ear as the adaptors or to the contralateral ear and were classified according to initial consonant ([b] or [d]). Essentially, performance for the primary targets during adaptation was impaired in the divided (dichotic) attention condition. Furthermore, secondary-target identification performance was better for the ear that was also monitored for the primary targets. This finding was interpreted in terms of attention-limited phonetic processing in the dichotic condition. With respect to aftereffects of adaptation, these were only found when adaptors and the subsequent test stimuli were presented to the same ear. This was interpreted in terms of a low acoustic level of adaptation. Importantly, the observed aftereffects were equivalent in magnitude for the monaural and dichotic adaptation conditions - that is, irrespective of focused (monaural) or divided (dichotic) attention. Hence, Mullennix (1986) concluded that adaptation takes place at an early acoustic level, irrespective of spatial attention, while the target identification task involves capacity-limited processes at a later phonetic stage.

Sussman (1993) used a slightly different design in which selective attention to [da] adaptors presented to one ear was manipulated by a classification task for [si] or [ $[\mathrm{i}]$ distractor syllables presented to the contralateral ear. As compared to passive adaptation to monaurally presented [da] syllables without [si] or [ji] distractors, Sussman even observed a trend toward greater aftereffects in the presence of attended distractors. She therefore confirmed Mullennix's (1986) findings: namely, that aftereffects are not impeded by directing attention away from adaptors. Samuel and Kat (1998) noted that Sussman's results could have been obscured by simultaneous adaptation to distractor syllables. Note that the attention conditions in Sussman's study differed acoustically, in that the distractor syllables were either present (dichotic condition) or absent (monaural condition). Samuel and Kat circumvented this problem by using visual distractors and found that aftereffects on a [ba]-[wa] continuum were unaltered by crossmodal attention to the distractors. Thus, they arrived at a conclusion similar to those of Sussman and Mullennix: namely, that attention to auditory adaptors is no prerequisite for speech aftereffects. However, while in Sussman's study selective attention was confounded with the presence of distractors, it is unclear whether the Samuel and Kat findings are restricted to situations in which the distractors are visual. To summarize, the reported studies suggest that speech aftereffects result from early auditory processing and do not require spatial attention.

At variance with these findings from phonetic perception, there is mounting evidence that selective attention modulates other adaptation-induced aftereffects. For instance, in vision, face identity aftereffects disappear when adaptors are unattended because participants are engaged in a digit memory task during adaptation (Moradi, Koch, \& Shimojo, 2005, Exp. 3, and Adams, Gray, Garner, \& Graf, 2010, for different results on adaptation to facial expressions). Visual adaptation to low-level features is also attention-dependent, such that inattention to adaptors reduces aftereffects (Chaudhuri, 1990; Suzuki \& Grabowecky, 2003). With respect to audition, selective attention appears to modulate the aftereffects of adaptation to simple tone stimuli. Specifically, one study has reported reduced auditory aftereffects to amplitude modulations in sinusoidal tones when attention was shifted away from tone adaptors presented to one channel, toward speech stimuli in the other channel (Kawashima, 2009). These findings, although obtained with only a small number of participants $(n=9)$, may indicate that unattended sounds are not fully processed under dichotic listening conditions.

At present, hardly any research has investigated the role of attention in auditory adaptation, and it is completely unclear whether attention modulates high-level auditory adaptation to nonlinguistic voice properties. However, on the basis of studies indicating that voices are processed preattentively during passive listening to one vocal stream (Beauchemin et al., 2006; Titova \& Näätänen, 2001), and despite spatial attention to a contralateral vocal stream in dichotic listening (Cherry, 1953; Treisman, 1964), one might expect that voice aftereffects should occur independently of spatial attention toward adaptors. Similarly, from studies suggesting that voice information is processed despite selective attention to speech content (e.g., Geiselman \& Bellezza, 1976; Kaganovich, Francis, \& Melara, 2006; Knösche, Lattner, Maess, Schauer, \& Friederici, 2002; Mullennix \& Pisoni, 1990), adaptation to voice gender might also proceed independently of dimensional attention to gender.

Here, we combined auditory adaptation with selectiveattention tasks during dichotic listening, to investigate (1) whether the perceptual aftereffects of adaptation to voice gender depend on spatial attention to gender-specific (male or female) adaptor voices (Exp. 1), and (2) whether voice gender aftereffects depend on dimensional attention to gender information in adaptor voices (Exp. 2). While spatial attention refers to different channels that can be selectively attended (left or right), dimensional attention refers to the 
kind of attended information within the attended channel (voice gender or syllable). During adaptation, our listeners simultaneously heard two streams of vowel-consonantvowel (VCV) syllables, with gender-specific (male or female) voices in one ear and gender-neutral (androgynous) voices in the contralateral ear. The androgynous voices, created by auditory morphing between veridical genderspecific voices (Kawahara \& Matsui, 2003), were conceived as neutral adaptors with no potential to induce adaptation, as they should not systematically activate gender-specific representations. A previous study using the same androgynous voices as adaptors had indicated that the category boundary for subsequent test voice perception was intermediate between the boundaries caused by purely male and female voice adaptors (Schweinberger et al., 2008).

Unlike a previous study on auditory speech adaptation (Sussman, 1993), we manipulated attention to adaptors under identical acoustic conditions, to dissociate top-down, attentional effects from bottom-up, stimulus-driven effects. During adaptation, we instructed participants to direct attention always to the same ear and to classify speaker gender (male or female; Exp. 1) or uttered syllable (/aba/ or /aga/; Exp. 2) while ignoring the contralateral ear. Aftereffects in voice gender perception were assessed via subsequent gender-morphed test voices presented binaurally.

\section{Method}

\section{Participants}

A group of 16 participants (eight male, eight female; mean age $=$ 23.3 years, range $=20-33$; all right-handed) contributed data to Experiment 1. A different group of 16 participants (eight male, eight female; mean age $=23.9$ years, range $=20-32 ; 13$ righthanded) contributed data to Experiment 2. All were unfamiliar with the voices used, and none reported otological problems. All but one of the participants (Exp. 1) were native German speakers. Six additional participants (four from Exp. 1 and two from Exp. 2) were excluded due to insufficient task compliance ( $>30 \%$ missing responses). The participants received $€ 5$ or course credit and gave written informed consent. The research was approved by the Faculty Ethics Committee of the University of Jena.

\section{Stimuli}

The stimuli were the voices of four male and four female speakers (20-27 years of age) from an earlier study (Zäske et al., 2009) uttering the VCV syllables /aba/, /aga/, /ibi/, and / igi/. These stimuli (16-bit resolution, $44.1 \mathrm{kHz}$ ) had been rootmean-square normalized and standardized with respect to overall duration $(686 \mathrm{~ms})$ and timing of the mean stop- consonant release (394 ms) by inserting and deleting periods in the center of vowels with Adobe Audition 1.5 software. Male and female voices were used as gender-specific adaptors. Androgynous adaptors and test voices had been created by auditory morphing between four original male/female speaker pairs for each syllable. Morphing had been performed via bilinear time-frequency transformation after selecting characteristic anchor points in the respective male and female spectrograms (Kawahara \& Matsui, 2003).

To create dichotic adaptor stimuli, preprocessed voices uttering /aba/ and /aga/ were combined, such that one channel contained an androgynous voice $(50 \% / 50 \%$ male/female morph) and the other channel contained either a male or a female voice. The adaptor sound files contained either the same syllables in both channels (/aba/-/aba/ or /aga/-/aga/) or different syllables (/aba/-/aga/ or /aga/-/aba/). Within a given adaptor sound file, speaker identities did not overlap. For instance, an androgynous voice of Speaker Pair 1 was combined with the female or male voice of Speaker Pair 2, 3, or 4. Combinations of speaker identities, voice genders, and syllables were counterbalanced across adaptor stimuli. The test stimuli were voice morphs of /ibi/ or /igi/ syllables of five different morph levels (MLs), with female/male proportions of $20 \% / 80 \%, 40 \% / 60 \%, 50 \% / 50 \%, 60 \% / 40 \%$, and $80 \% /$ $20 \%$, and with identical information in both channels (binaural presentation). Voices were presented at a peak intensity of approximately $66 \mathrm{~dB}(\mathrm{~A})$, as measured with a Brüel \& Kjær Precision Sound Level Meter, Type 2206. Table 1 shows the fundamental frequencies $(f 0)$ and formant frequencies $(F 1$, $F 2$, and $F 3$ ) for the male (ML $0 \% / 100 \%$ ), androgynous (ML $50 \% / 50 \%$ ), and female (ML $100 \% / 0 \%$ ) syllable stimuli, as determined with Praat software (Boersma \& Weenink, 2012).

\section{Procedure}

The participants were tested individually in a soundattenuated chamber. Instructions were delivered via the computer screen, and the experimenter did not talk to participants during the session, to avoid spurious voice adaptation effects.

At the beginning of the experiment, the participants were instructed to attend to the left or the right ear whenever a green fixation cross appeared along with two simultaneous voices (cf. Fig. 1). For a given participant, the attended channel remained the same throughout the entire experiment. In each trial, three dichotic voice adaptors were followed by a single test voice that was presented binaurally. For attended adaptors, the participants classified either voice gender (Exp. 1) or the uttered syllable (Exp. 2). The test voices were classified according to gender in both experiments.

The participants performed two adaptation blocks of 192 trials each. Depending on the adaptation block, the dichotic adaptors were composed of a male voice paired with an 
Table 1 Frequency analysis for the syllable stimuli from each speaker pair (P) at three selected morph levels (MLs), as measured in the stable phase of the first vowel (50-200 ms after vowel onset)

\begin{tabular}{|c|c|c|c|c|c|c|c|c|c|c|c|c|c|c|c|c|c|}
\hline & \multirow[b]{2}{*}{ ML in $\%$} & \multicolumn{4}{|c|}{$f 0[\mathrm{~Hz}]$} & \multicolumn{4}{|c|}{$F 1[\mathrm{~Hz}]$} & \multicolumn{4}{|c|}{$F 2[\mathrm{~Hz}]$} & \multicolumn{4}{|c|}{$F 3[\mathrm{~Hz}]$} \\
\hline & & aba & aga & ibi & igi & aba & aga & ibi & igi & aba & aga & ibi & igi & aba & aga & ibi & igi \\
\hline \multirow[t]{3}{*}{ P1 } & $0 / 100$ & 111 & 110 & 125 & 118 & 732 & 748 & 293 & 311 & 1138 & 1178 & 2245 & 2249 & 2553 & 2502 & 3093 & 3071 \\
\hline & $50 / 50$ & 155 & 153 & 171 & 164 & 766 & 764 & 379 & 358 & 1240 & 1246 & 2433 & 2360 & 2622 & 2570 & 3118 & 3116 \\
\hline & $100 / 0$ & 216 & 214 & 235 & 229 & 833 & 795 & 460 & 457 & 1385 & 1576 & 2644 & 2683 & 3171 & 3044 & 3718 & 3635 \\
\hline \multirow[t]{3}{*}{ P2 } & $0 / 100$ & 111 & 125 & 158 & 171 & 755 & 765 & 300 & 282 & 1218 & 1262 & 2316 & 2273 & 2706 & 2571 & 3261 & 3212 \\
\hline & $50 / 50$ & 153 & 160 & 186 & 201 & 805 & 790 & 396 & 366 & 1273 & 1276 & 2444 & 2424 & 2742 & 2595 & 3693 & 3299 \\
\hline & $100 / 0$ & 212 & 207 & 222 & 230 & 1035 & 996 & 412 & 399 & 1546 & 1585 & 3002 & 2798 & 3189 & 3174 & 3840 & 3767 \\
\hline \multirow[t]{3}{*}{ P3 } & $0 / 100$ & 116 & 118 & 124 & 127 & 698 & 693 & 258 & 256 & 1045 & 1051 & 2235 & 2201 & 2470 & 2330 & 3268 & 3053 \\
\hline & $50 / 50$ & 166 & 164 & 172 & 176 & 769 & 866 & 342 & 354 & 1181 & 1522 & 2286 & 2334 & 2523 & 2424 & 3326 & 3162 \\
\hline & $100 / 0$ & 236 & 229 & 238 & 242 & 830 & 913 & 478 & 468 & 1353 & 1591 & 2583 & 2798 & 2803 & 2785 & 3871 & 3893 \\
\hline \multirow[t]{3}{*}{ P4 } & $0 / 100$ & 111 & 109 & 121 & 130 & 717 & 748 & 368 & 331 & 1251 & 1336 & 2500 & 2381 & 2502 & 2363 & 3438 & 3458 \\
\hline & $50 / 50$ & 153 & 150 & 166 & 166 & 777 & 813 & 396 & 346 & 1333 & 1443 & 2583 & 2662 & 2648 & 2687 & 3528 & 3548 \\
\hline & $100 / 0$ & 210 & 207 & 224 & 214 & 846 & 889 & 446 & 387 & 1427 & 1469 & 2647 & 2690 & 2703 & 2788 & 3651 & 3659 \\
\hline
\end{tabular}

androgynous voice $(M / A)$ or a female voice paired with an androgynous voice $(F / A)$. Adaptation blocks were further subdivided in two blocks of 96 randomized trials, depending on whether participants were attending to gender-specific or androgynous adaptors. The order of adaptation blocks and the attended ear were counterbalanced across male and female participants in both experiments, as were the assignments of adaptor speakers to ears. Morphed test voices with female/male proportions of $20 \% / 80 \%, 40 \% / 60 \%, 50 \% /$ $50 \%, 60 \% / 40 \%$, and $80 \% / 20 \%$ were presented according

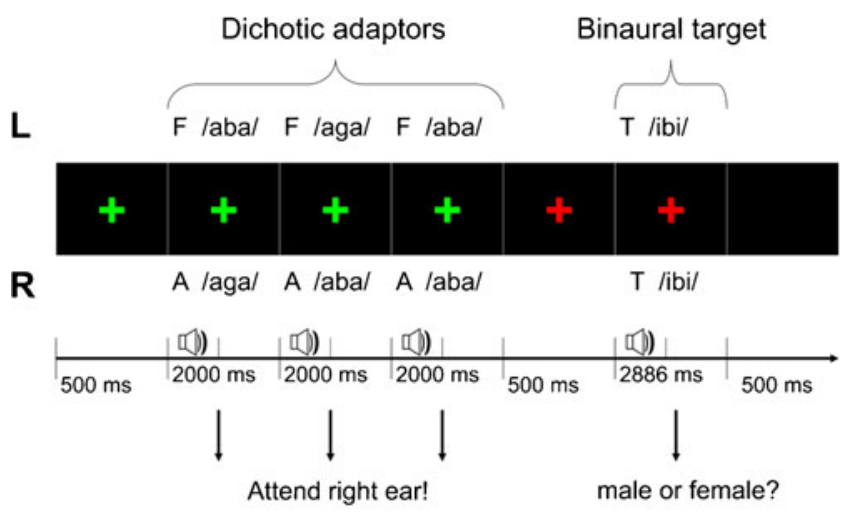

Exp. 1 male or female?

Exp. 2 aba or aga?

Fig. 1 Trial procedure in Experiments 1 and 2. Participants simultaneously adapted to either female and androgynous voices (F/A, as in this example, with gender-specific adaptors in the left ear) or male and androgynous voices (M/A), presented dichotically - that is, via simultaneous presentation in the left $(\mathrm{L})$ and right $(\mathrm{R})$ channels. They selectively attended to gender-specific (female or male) or androgynous adaptors and classified either the voice gender (Exp. 1) or spoken syllable (Exp. 2). Subsequent test voices (T), presented binaurally, were always classified according to gender to the method of constant stimuli. For a given trial, the adaptor and test voices always originated from different speaker pairs, and simultaneous dichotic adaptors also always originated from different speaker pairs. For instance, if a test voice was a morph from Speaker Pair 4, the dichotic adaptors originated from Pairs 1, 2, and 3 to minimize potential low-level adaptation effects.

Trials started with a green fixation cross for $500 \mathrm{~ms}$, joined by three consecutive dichotic adaptors $(886 \mathrm{~ms}$ each, including $100 \mathrm{~ms}$ of silence at the beginning and the end). For each of the three dichotic adaptors, participants decided whether the voices in the designated ear (left or right) were male or female (Exp. 1) or whether the spoken syllable was / aba/ or /aga/ (Exp. 2). While adaptor gender varied blockwise, the adaptor syllables varied randomly within each trial. From adaptor onset, participants had 2,000 ms to enter their response until the next dichotic adaptor was presented. Following the third adaptor, the fixation cross turned red to indicate the upcoming test voice, which was presented $500 \mathrm{~ms}$ thereafter. Participants were instructed to classify the gender (male or female) of the binaural test voice as quickly and accurately as possible. For responses slower than 2,886 ms (duration of the sound file plus the 2,000$\mathrm{ms}$ response window) following test voice onset, the response was not recorded and feedback $(500 \mathrm{~ms})$ was given: "Please respond faster." Otherwise, a blank screen appeared for $500 \mathrm{~ms}$. Thus, the total trial duration was $10,386 \mathrm{~ms}$ (cf. Fig. 1). All responses were given via a computer keyboard by pressing the "a" or the "l" key. These keys represented the "male" and "female" response categories during adaptation in Exp. 1, and during test voice assessment in both experiments. Due to the task switch in Exp. 2, these keys additionally served to enter /aba/ or /aga/ responses to the 
attended adaptors. The assignments of response categories to keys were counterbalanced across participants. The main experiments were preceded by 16 practice trials with voices from a fifth speaker pair that was not used in the experimental trials.

\section{Results}

Errors of omission and responses $<200 \mathrm{~ms}$ from sound onset were excluded ( $3.7 \%$ of responses to adaptors, and $1.6 \%$ of responses to test voices). For each experiment, voice gender aftereffects in test voice perception were analyzed using analyses of variance (ANOVAs) with repeated measures on adaptation condition, attended adaptor, and morph levels, with data collapsed across attended ears and participant genders. We considered the participant sample too small for an analysis of the between-subjects factors Attended Ear and Participant Gender. In Experiment 1, gender classification during adaptation was highly accurate $(99.6 \%$ correct) for gender-specific adaptors, suggesting that adaptors were selectively attended and/or that the task was very easy. As expected, gender classification was balanced for androgynous adaptors ( $54.3 \%$ female responses), confirming that these stimuli were perceived as being neither male nor female. For test voices, a significant voice gender aftereffect (VGAE) was reflected in more female classifications when test stimuli were preceded by $\mathrm{M} / \mathrm{A}$ as compared to $\mathrm{F} / \mathrm{A}$ adaptors, $F(1,15)=8.52, p=$ $.011, \eta_{p}^{2}=.362$. Importantly, we obtained an interaction of adaptation condition (F/A vs. M/A) and attended adaptor (gender-specific vs. gender-neutral), $F(1,15)=10.20, p=$ $.006, \eta_{p}^{2}=.405$. As expected, a main effect of morph level also emerged, $F(4,60)=146.51, p<.001, \eta_{p}^{2}=.907$. Likewise, an interaction between adaptation condition and morph level, $F(4,60)=3.27, p=.017, \eta_{p}^{2}=.179$, reflected the fact that adaptation effects tended to be smaller for the less ambiguous test voices (see Fig. 2a).

To follow up on the interaction between adaptation condition and attended adaptors, we performed separate analyses for both attended adaptor conditions. A significant VGAE emerged only when gender-specific adaptors had been attended, $F(1,15)=$ $14.65, p=.002, \eta_{p}^{2}=.494$, and the VGAE was slightly smaller for less ambiguous morph levels: interaction of adaptation condition and ML, $F(4,60)=2.65, p=.042, \eta_{p}^{2}=.150$. By contrast, no VGAE was present when gender-neutral adaptors had been attended, $F(1,15)=0.21, p=.657, \eta_{p}^{2}=.014$, and no interaction occurred between adaptation condition and ML, $F(4,60)=1.91, p=.121, \eta_{p}^{2}=.113$; compare Fig. 2a and b.

Although our study did not include a preadaptation baseline, we evaluated the aftereffects caused by attended male and female adaptor voices with reference to the respective attend-to-androgynous adaptor conditions. These analyses revealed that adaptation to male voices caused test voices to be perceived as more female ( $M=41.1 \%$ vs. $34.8 \%), F(1,15)=$ $8.33, p=.011, \eta_{p}^{2}=.357$, whereas adaptation to female voices only caused a numerical decrement in female judgments $(M=$ $31.4 \%$ vs. $33.9 \%), F(1,15)=1.50, p=.239, \eta_{p}^{2}=.091$.

In order to test whether the VGAE requires selective dimensional attention to gender in adaptors, Experiment 2 required syllable classification (/aba/ vs. /aga/) during adaptation. Note that, apart from the task difference during adaptation, both experiments involved physically identical stimuli. Syllable classification was relatively accurate (84.6\% and $81.6 \%$ correct for gender-specific and androgynous adaptors, respectively), indicating a successful manipulation of attention. Test voices were perceived as being increasingly female with increasing $\mathrm{ML}, F(4,60)=105.66$, $p<.001, \eta_{p}^{2}=.876$, but no significant interactions emerged of ML with any other variables, all $F$ values $<1.29$. The main effects of adaptation condition $[F(1,15)=13.12, p=.003$, $\left.\eta_{p}^{2}=.466\right]$ and attended adaptor $[F(1,15)=9.93, p=.007$, $\left.\eta_{p}^{2}=.398\right]$ were further qualified by an interaction $[F(1,15)=$ $\left.5.76, p=.03, \eta_{p}^{2}=.277\right]$. As before, the VGAE was significant following spatial attention to gender-specific voices, even when dimensional attention was directed to speech content, $F(1,15)=21.4, p<.001, \eta_{p}^{2}=.588$. Again, a VGAE was absent after attention to androgynous voices, $F(1,15)<1$; compare Fig. $2 \mathrm{c}$ and $\mathrm{d}$.

An additional evaluation of the VGAE with respect to attend-to-androgynous adaptor conditions revealed significantly more female responses following attention to male adaptors $(45.7 \%)$ than following androgynous adaptors $(38.0 \%)\left[F(1,15)=15.09, p=.001, \eta_{p}^{2}=.502\right]$, but no difference following attention to female $(37.2 \%)$ versus androgynous $(37.5 \%)$ adaptors $[F(1,15)<1]$.

Finally, we compared performance in both experiments via an ANOVA that included the between-subjects factors Task, Attended Ear, and Listener Gender, with repeated measures on adaptation condition and attended adaptor, and with data collapsed across MLs. We found no effects involving task or ear, confirming that aftereffects were independent of task and laterality. However, female listeners perceived test voices as being slightly more male $(31.1 \%$ female responses) than did the male listeners ( $43.6 \%$ female responses) overall, $F(1,24)=5.92, p=.023, \eta_{p}^{2}=.198$, a finding that might tentatively be attributed to continuous contrastive adaptation to one's own voice. Interestingly, listener gender modulated the interaction of adaptation condition and attended adaptor, $F(1,24)=8.83, p=.007, \eta_{p}^{2}=$ .269. In short, a stronger VGAE was seen in male than in female listeners after attending to gender-specific adaptors (see Fig. 3). Two separate ANOVAs for attended gender-specific adaptors and attended androgynous adaptors revealed interactions of adaptation condition and listener gender for the attended gender-specific adaptors, $F(1,24)=11.34, p=.003, \eta_{p}^{2}=$ .321 , but not for attended androgynous adaptors $(p>.05)$. 
Fig. 2 Percentage of "female" classifications for five morph levels of test voices after attending to voice gender (Exp. 1) in gender-specific (panel a) or androgynous (panel b) adaptors, and after attending to spoken syllable (Exp. 2) in genderspecific (panel c) and androgynous (panel d) adaptors. (Top) Clear voice gender aftereffects (VGAEs) were observed when gender-specific

( $\mathrm{F}$ or $\mathrm{M}$ ) adaptors had been spatially attended (panel a), but were absent following attention to androgynous (a) voices (panel b). (Bottom) VGAEs were preserved when syllables were attended in genderspecific adaptors (panel c), but were absent following attention to syllables in androgynous adaptors (panel d). Thus,

VGAEs require selective spatial, but not dimensional attention. Error bars show standard errors of the means (SEMs)

\section{Exp. 1) Attention to Gender}
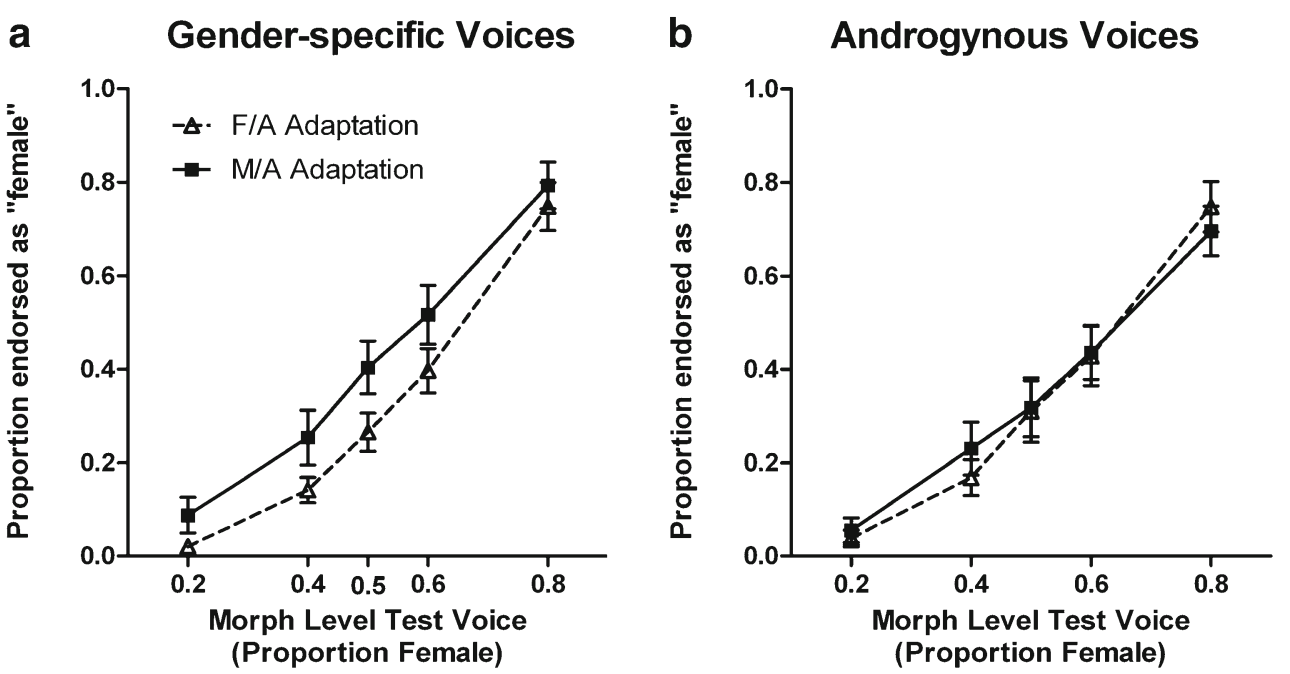

\section{Exp. 2) Attention to Syllable}
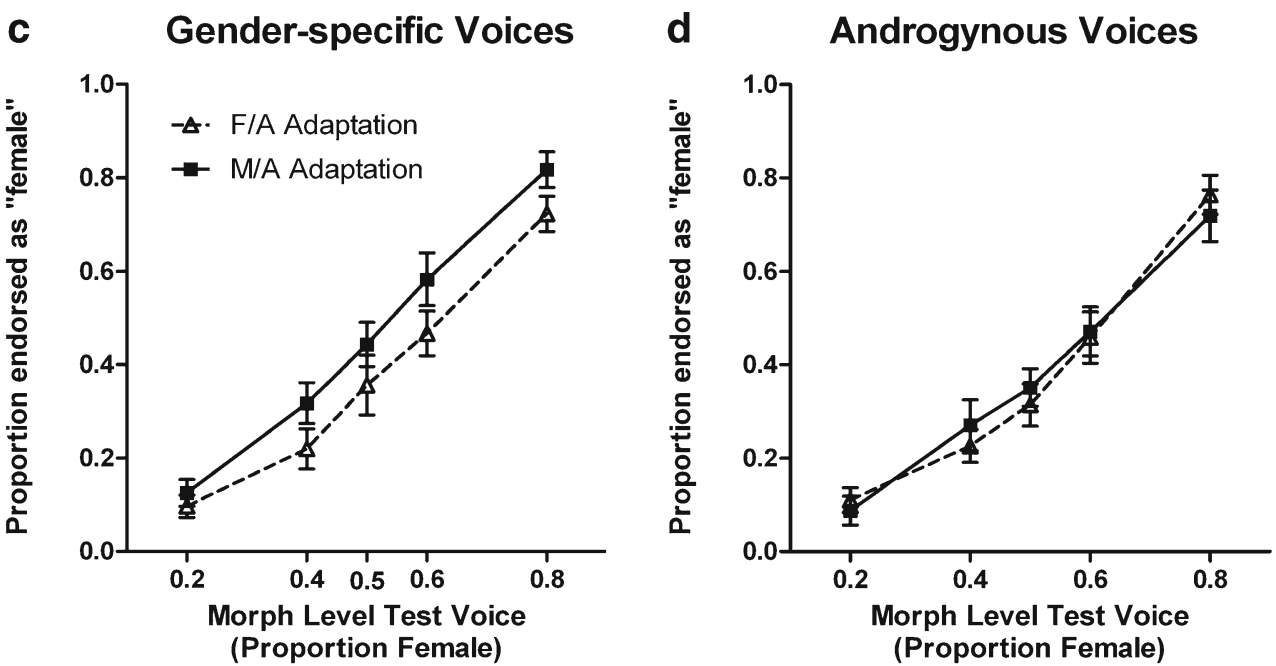

Importantly, the VGAE following attended genderspecific adaptors was significant for both listener groups:

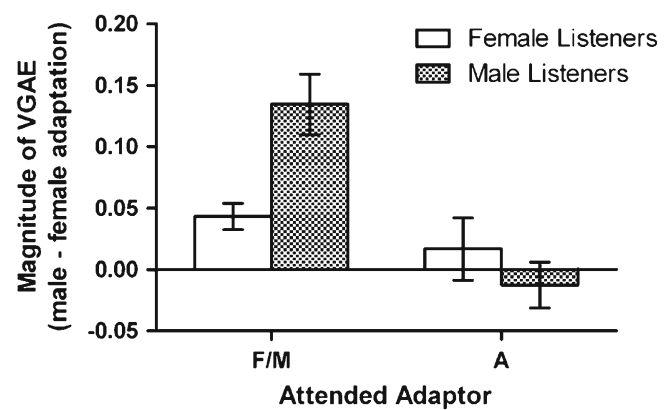

Fig. 3 Magnitude of voice gender aftereffects (M/A minus F/A adaptation) after attending gender-specific female or male (F/M) versus androgynous (A) adaptors, as a function of listener gender. Error bars show standard errors of the means (SEMs) $t(15)=-5.46, p<.001$, and $t(15)=-4.0, p<.001$, for male and female listeners, respectively.

\section{Discussion}

Our results demonstrate for the first time that voice aftereffects are disrupted by spatial inattention to adaptor voices. Specifically, under identical acoustic conditions, the VGAE was elicited when attention was focused on the ear containing gender-specific (male or female) adaptors, but not when attention was focused on gender-neutral (androgynous) adaptors in the contralateral ear. Accordingly, this modulation is purely top-down-driven, reminiscent of findings from visual adaptation that face aftereffects depend on attention to the adaptor (Moradi et al., 2005). Our findings are also in 
line with attention-dependent auditory aftereffects following adaptation to simple tone stimuli (Kawashima, 2009). As in the present study, Kawashima used dichotic listening to spatially separate amplitude-modulated tone adaptors from speech stimuli and found reduced aftereffects from tones when attention was directed at speech stimuli rather than at tones. However, while a direct comparison between studies may be complicated by differences in the relative spectral complexity and salience of simultaneously presented sounds in the present and in Kawashima's study, results from both studies support the notion that spatially unattended sounds are not processed fully under dichotic listening conditions.

At first glance, this result could appear to be at odds with other findings that task-irrelevant voice gender is processed not only when one voice is presented at a time (Fenn et al., 2011), but also during dichotic listening to two speakers simultaneously (Cherry, 1953; Treisman, 1964). However, we do not see a strong contradiction with those findings. With respect to Cherry, a crucial difference between the studies could be that our experiments did not involve unexpected changes in voice gender (due to our block-wise variation of adaptor gender). Thus, while voice gender may be salient and capture attention (Fenn et al., 2011), such attentional capture may occur only in the case of unexpected changes in voice gender. One could still argue that Treisman's finding of intrusions from an unattended message during shadowing of a contralateral message indicates that voice information in the unattended channel is processed to some extent. Crucially, she found that this was particularly so for two similar (female) voices, but was less likely for two dissimilar (male and female) voices. Treisman argued that voice information can be rejected at an early processing stage if the two voices are acoustically dissimilar. In other words, stream segregation based on voice quality is a prerequisite for successful suppression of task-irrelevant input. In the present study, high performance in the syllable classification task (Exp. 2) indicated that the adaptors were sufficiently dissimilar to be perceived as originating from two different sources. While this cannot be directly tested for the gender classification task (Exp. 1), due to the predictability of adaptor gender in the male and female adaptation blocks, we assume that the dichotic adaptors in Experiment 1 were as well discriminable as those in Experiment 2, since we used identical stimuli.

How do our findings relate to findings that speechinduced aftereffects in the perception of linguistic features occur automatically - that is, even when spatial attention is shifted away from adaptors (Mullennix, 1986; Sussman, 1993)? The previous authors have argued that while speech aftereffects are due to attention-independent low-level adaptation, later (phonetic) stages of speech processing are affected by limited attentional capacities in dichotic listening. On the basis of this reasoning, and assuming that early linguistic and nonlinguistic processing occurs in parallel (Knösche et al., 2002), the present lack of voice aftereffects following spatial inattention to adaptors could be further evidence for higher-level perceptual gender representations underlying the VGAE (Schweinberger et al., 2008). Accordingly, activation of these gender representations could be more dependent on spatial attention, as compared with the preceding lower-level stages of analysis.

This may seem to be at odds with findings that nonlinguistic information is processed preattentively, as indexed by the MMN (Beauchemin et al., 2006; Titova \& Näätänen, 2001). However, these results may be specific to situations in which one vocal stream is presented at a time. By contrast, during dichotic listening to simple tones, the MMN for deviants in the unattended ear is reduced, relative both to the attended ear and to passive listening during reading (Alain \& Woods, 1997; Näätänen, Paavilainen, Tiitinen, Jiang, \& Alho, 1993), which is known to involve voice imagery (Alexander \& Nygaard, 2008). With respect to speech or voice adaptation effects, it is thus possible that spatial attention modulates the neural adaptability of higher-level perceptual representations and/or of lower-level neurons connected to these.

In order to understand how adaptation and attention may jointly affect voice perception on a neuronal level, it needs to be considered that both are known to modulate neuronal responses. However, while adaptation attenuates neuronal response (Grill-Spector, Henson, \& Martin, 2006; Jääskeläinen, Ahveninen, Belliveau, Raij, \& Sams, 2007), attention increases it (Jääskeläinen et al., 2007; Petkov et al., 2004). For instance, auditory evoked potentials (AEPs) are attenuated by repetition of both vowels (Kuriki, Ohta, \& Koyama, 2007) and voices (Schweinberger et al., 2011; Zäske et al., 2009). Functional imaging studies on voice adaptation and voice learning have located repetition-induced reductions of brain activity within inferior frontal (Latinus \& Belin, 2011) and anterior temporal (Andics et al., 2010; Belin \& Zatorre, 2003) lobe areas that may be voice-selective (Belin, Zatorre, Lafaille, Ahad, \& Pike, 2000). Unlike adaptation, selective attention enhances processing, as reflected in both early AEPs (for a review, see Fritz, Elhilali, David, \& Shamma, 2007) and fMRI studies. For instance, attention to voice identity, irrespective of speech content, activates voice-selective areas in right anterior superior temporal sulcus (von Kriegstein, Eger, Kleinschmidt, \& Giraud, 2003).

Despite this proximity of areas activated by both adaptation and selective attention to voices, the precise neurophysiological underpinnings are unclear. Adaptation could be due to various mechanisms, including neuronal fatigue, facilitation, or sharpening (Grill-Spector et al., 2006; Jääskeläinen et al., 2011). Similarly, auditory selective attention could involve two possibly complementary mechanisms (Kauramäki, Jääskeläinen, \& Sams, 2007; Neelon, 
Williams, \& Garell, 2011; Okamoto, Stracke, Wolters, Schmael, \& Pantev, 2007), of attentional gain (i.e., enhanced neuronal activity for attended objects) and neuronal sharpening (i.e., receptive field changes). As a result, both adaptation and attention may enhance the feature selectivity of neurons, and thus sensitivity for stimulus change. Accordingly, it has been proposed that attention could regulate adaptation by increasing neuronal adaptability (Boynton, 2004).

This notion appears to be reconcilable with our results, although at present we can only speculate about the precise neuronal mechanisms underlying our behavioral effects. Accordingly, selective spatial attention to gender-specific voices may enhance the adaptability of neurons representing voice gender. Averting spatial attention from gender-specific adaptors, by contrast, may prevent sufficient analysis, thereby suppressing adaptation.

Strikingly, whereas voice adaptation was completely abolished under spatial inattention, adaptation was fully preserved under dimensional inattention (Exp. 2). Specifically, attention to a phonetic dimension (syllable identity, as defined by place of articulation) elicited VGAEs virtually identical to those resulting from attention to voice gender (Exp. 1). This is in line with studies showing that the processing of nonlinguistic voice information proceeds despite selective attention to speech content (Geiselman \& Bellezza, 1976; Kaganovich et al., 2006; Knösche et al., 2002; Mullennix \& Pisoni, 1990). On the one hand, this finding corroborates the notion that voice quality is processed preattentively; on the other, our results establish important limits of the preattentive processing of voice quality, insofar as spatial inattention abolished voice adaptation in both experiments.

It may be noted that the androgynous $(50 \% / 50 \%)$ test voices in the present study were classified as male slightly more frequently than they were classified as female (37.4\%). It should be noted that this does not appear to be a consequence of speaker selection, because androgynous adaptor voices, generated from the same speaker continua and using the same morph algorithm, did not show this asymmetry (54.3\% female classifications). One obvious difference is that the test voices were /ibi/ and /igi/ tokens, whereas the adaptors comprised /aba/ and /aga/ tokens: It is possible that gender perception of androgynous voices is influenced by the kind of vowel, since females have been reported to have a larger acoustic vowel space (e.g., Simpson \& Ericsdotter, 2007), and since there is evidence for a degree of interdependence between vowel and gender perception (Eklund \& Traunmüller, 1997). Although the present "male bias" in the test voices is largely irrelevant for our main findings, it could perhaps explain why female adaptors appeared to be less efficient than male adaptors in eliciting contrastive aftereffects, in a situation in which test voices already tended to be perceived as male.
An unexpected finding was that male listeners were more adaptable to voice gender than were female listeners, a finding that is at variance with our previous studies that did not indicate gender differences in voice adaptability (Schweinberger et al., 2008; Zäske et al., 2009). Although gender differences have been reported previously, both for auditory adaptability to low-level features (D'Alessandro \& Norwich, 2009) and for attention to auditory sequences, as reflected in early AEPs (Nagy, Potts, \& Loveland, 2003), a relation to the present results remains speculative at this point. However, if attention increases adaptability, men might have been more adaptable to attended voices because they more easily focused on task-relevant information while ignoring irrelevant information. Related to this, an eventrelated potential study found evidence for decreased automatic processing of irrelevant prosodic information in men, as compared to women (Schirmer \& Kotz, 2003). Overall, the present gender differences may therefore predominantly reflect differences in selective attention, rather than in voice gender adaptability per se. Voice adaptation was here observed after simultaneous exposure to more than one voice, with the spatial location of voices as a cue to stream segregation. A question for future research may therefore be whether similar effects would emerge when the attended "channel" is defined by other, nonspatial dimensions, such as emotional intonation or accent. This may be important, as selective attention to spatial versus nonspatial information preferentially recruits auditory "where" versus "what" pathways in the brain, respectively (Arnott, Binns, Grady, \& Alain, 2004).

It has been argued that adaptation effects in speech perception may be due to anchoring effects such as auditory contrast (e.g., Diehl, Lang, \& Parker, 1980), instead of to sensory fatigue. In the present study, an alternative explanation in terms of response bias was largely excluded, because adaptation was also observed following a task that did not require gender processing, but rather phonetic processing, of the adaptors (Exp. 2). We believe that due to the task switch between adaptation and test, the syllable classification task during adaptation is unlikely to have changed the response criterion for subsequent gender classification.

Taken together, our results support the notion that if two voices are presented simultaneously, spatial attention to one voice prevents processing of a concurrent voice in the contralateral ear, thereby abolishing voice adaptation. Once spatial attention is directed to a voice, however, preserved adaptation effects suggest automatic processing of speaker gender, even when it is irrelevant for the task at hand. In conclusion, voice adaptation requires spatial, but not dimensional, selective attention.

Author note This research was supported by a grant from the Deutsche Forschungsgemeinschaft (DFG-GZ Schw 511/10-1) to S.R.S. 


\section{References}

Adams, W. J., Gray, K. L. H., Garner, M., \& Graf, E. W. (2010). Highlevel face adaptation without awareness. Psychological Science, 21, 205-210. doi:10.1177/0956797609359508

Alain, C., \& Woods, D. L. (1997). Attention modulates auditory pattern memory as indexed by event-related brain potentials. Psychophysiology, 34, 534-546.

Alexander, J. D., \& Nygaard, L. C. (2008). Reading voices and hearing text: Talker-specific auditory imagery in reading. Journal of Experimental Psychology. Human Perception and Performance, 34, 446-459.

Andics, A., McQueen, J. M., Petersson, K. M., Gal, V., Rudas, G., \& Vidnyanszky, Z. (2010). Neural mechanisms for voice recognition. NeuroImage, 52, 1528-1540.

Anstis, S., Verstraten, F. A. J., \& Mather, G. (1998). The motion aftereffect. Trends in Cognitive Sciences, 2, 111-117.

Arnott, S. R., Binns, M. A., Grady, C. L., \& Alain, C. (2004). Assessing the auditory dual-pathway model in humans. NeuroImage, 22, 401-408.

Beauchemin, M., De Beaumont, L., Vannasing, P., Turcotte, A., Arcand, C., Belin, P., \& Lassonde, M. (2006). Electrophysiological markers of voice familiarity. European Journal of Neuroscience, 23, 30813086. doi:10.1111/j.1460-9568.2006.04856.x

Belin, P., \& Zatorre, R. J. (2003). Adaptation to speaker's voice in right anterior temporal lobe. NeuroReport, 14, 2105-2109.

Belin, P., Zatorre, R. J., Lafaille, P., Ahad, P., \& Pike, B. (2000). Voiceselective areas in human auditory cortex. Nature, 403, 309-312.

Bestelmeyer, P. E. G., Rouger, J., DeBruine, L. M., \& Belin, P. (2010). Auditory adaptation in vocal affect perception. Cognition, 117, 217-223.

Boersma, P., \& Weenink, D. (2012). Praat: Doing phonetics by computer (Version 5.3.16) [Computer program]. Retrieved 23 May, 2012, from www.praat.org

Boynton, G. M. (2004). Adaptation and attentional selection. Nature Neuroscience, 7, 8-10.

Charest, I., Pernet, C., Latinus, M., Crabbe, F., \& Belin, P. (in press). Cerebral processing of voice gender studied using a continuous carryover fMRI design. Cerebral Cortex. doi:10.1093/cercor/bhs090

Chaudhuri, A. (1990). Modulation of the motion aftereffect by selective attention. Nature, 344, 60-62. doi:10.1038/344060a0

Cherry, E. C. (1953). Some experiments on the recognition of speech, with one and with two ears. Journal of the Acoustical Society of America, 25, 975-979.

Cooper, W. E., \& Blumstein, S. E. (1974). Labial feature analyzer in speech perception. Perception \& Psychophysics, 15, 591-600.

D'Alessandro, L. M., \& Norwich, K. H. (2009). Loudness adaptation measured by the simultaneous dichotic loudness balance technique differs between genders. Hearing Research, 247, 122-127.

Diehl, R. L., Kluender, K. R., \& Parker, E. M. (1985). Are selective adaptation and contrast effects really distinct. Journal of Experimental Psychology. Human Perception and Performance, 11, 209-220.

Diehl, R. L., Lang, M., \& Parker, E. M. (1980). Further parallel between selective adaptation and contrast. Journal of Experimental Psychology. Human Perception and Performance, 6, 24-44.

Eimas, P. D., \& Corbit, J. D. (1973). Selective adaptation of linguistic feature detectors. Cognitive Psychology, 4, 99-109.

Eklund, I., \& Traunmüller, H. (1997). Comparative study of male and female whispered and phonated versions of the long vowels of Swedish. Phonetica, 54, 1-21.

Fenn, K. M., Shintel, H., Atkins, A. S., Skipper, J. I., Bond, V. C., \& Nusbaum, H. C. (2011). When less is heard than meets the ear: Change deafness in a telephone conversation. Quarterly Journal of Experimental Psychology, 64, 1442-1456.
Fritz, J. B., Elhilali, M., David, S. V., \& Shamma, S. A. (2007). Auditory attention-Focusing the searchlight on sound. Current Opinion in Neurobiology, 17, 437-455.

Geiselman, R. E., \& Bellezza, F. S. (1976). Long-term memory for speakers voice and source location. Memory \& Cognition, 4, 483489.

Grantham, D. W., \& Wightman, F. L. (1979). Auditory motion aftereffects. Perception \& Psychophysics, 26, 403-408.

Grill-Spector, K., Henson, R., \& Martin, A. (2006). Repetition and the brain: Neural models of stimulus-specific effects. Trends in Cognitive Sciences, 10, 14-23. doi:10.1016/j.tics.2005.11.006

Hillenbrand, J. M., \& Clark, M. J. (2009). The role of $f_{0}$ and formant frequencies in distinguishing the voices of men and women. Attention, Perception, \& Psychophysics, 71, 1150-1166. doi:10.3758/APP.71.6.1150

Jääskeläinen, I. P., Ahveninen, J., Andermann, M. L., Belliveau, J. W., Raij, T., \& Sams, M. (2011). Short-term plasticity as a neural mechanism supporting memory and attentional functions. Brain Research, 1422, 66-81.

Jääskeläinen, I. P., Ahveninen, J., Belliveau, J. W., Raij, T., \& Sams, M. (2007). Short-term plasticity in auditory cognition. Trends in Neurosciences, 30, 653-661.

Kaganovich, N., Francis, A. L., \& Melara, R. D. (2006). Electrophysiological evidence for early interaction between talker and linguistic information during speech perception. Brain Research, 1114, 161-172.

Kauramäki, J., Jääskeläinen, I. P., \& Sams, M. (2007). Selective attention increases both gain and feature selectivity of the human auditory cortex. PLoS One, 2, e909. doi:10.1371/journal.pone.0000909

Kawahara, H., \& Matsui, H. (2003). Auditory morphing based on an elastic perceptual distance metric in an interference-free timefrequency representation. In Proceedings of the 2003 IEEE International Conference on Acoustics, Speech, and Signal Processing (Vol. I, pp. 256-259). Piscataway: IEEE Press.

Kawashima, T. (2009). Attention modulates auditory adaptation produced by amplitude modulation. Journal of the Acoustical Society of America, 126, EL123-EL127.

Knösche, T. R., Lattner, S., Maess, B., Schauer, M., \& Friederici, A. D. (2002). Early parallel processing of auditory word and voice information. NeuroImage, 17, 1493-1503.

Kuriki, S., Ohta, K., \& Koyama, S. (2007). Persistent responsiveness of long-latency auditory cortical activities in response to repeated stimuli of musical timbre and vowel sounds. Cerebral Cortex, 17, $2725-2732$.

Latinus, M., \& Belin, P. (2011). Anti-voice adaptation suggests prototypebased coding of voice identity. Frontiers in Psychology, 2(175), 1-12.

Leopold, D. A., O’Toole, A. J., Vetter, T., \& Blanz, V. (2001). Prototype-referenced shape encoding revealed by high-level aftereffects. Nature Neuroscience, 4, 89-94. doi:10.1038/82947

Moradi, F., Koch, C., \& Shimojo, S. (2005). Face adaptation depends on seeing the face. Neuron, 45, 169-175. doi:10.1016/ j.neuron.2004.12.018

Morse, P. A., Kass, J. E., \& Turkienicz, R. (1976). Selective adaptation of vowels. Perception \& Psychophysics, 19, 137-143.

Mullennix, J. W. (1986). Attentional limitations in the perception of speech. Unpublished doctoral dissertation, State University of New York at Buffalo, Buffalo, NY.

Mullennix, J. W., Johnson, K. A., Topcu-Durgun, M., \& Farnsworth, L. M. (1995). The perceptual representation of voice gender. Journal of the Acoustical Society of America, 98, 3080-3095.

Mullennix, J. W., \& Pisoni, D. B. (1990). Stimulus variability and processing dependencies in speech-perception. Perception \& Psychophysics, 47, 379-390.

Näätänen, R., Paavilainen, P., Rinne, T., \& Alho, K. (2007). The mismatch negativity (MMN) in basic research of central auditory processing: A review. Clinical Neurophysiology, 118, 2544-2590. 
Näätänen, R., Paavilainen, P., Tiitinen, H., Jiang, D., \& Alho, K. (1993). Attention and mismatch negativity. Psychophysiology, 30, 436-450.

Nagy, E., Potts, G. F., \& Loveland, K. A. (2003). Sex-related ERP differences in deviance detection. International Journal of Psychophysiology, 48, 285-292.

Neelon, M. F., Williams, J., \& Garell, P. C. (2011). Elastic attention: Enhanced, then sharpened response to auditory input as attentional load increases. Frontiers in Human Neuroscience, 5, 41. doi:10.3389/fnhum.2011.00041

Okamoto, H., Stracke, H., Wolters, C. H., Schmael, F., \& Pantev, C. (2007). Attention improves population-level frequency tuning in human auditory cortex. Journal of Neuroscience, 27, 1038310390.

Petkov, C. I., Kang, X. J., Alho, K., Bertrand, O., Yund, E. W., \& Woods, D. L. (2004). Attentional modulation of human auditory cortex. Nature Neuroscience, 7, 658-663.

Samuel, A. G., \& Kat, D. (1998). Adaptation is automatic. Perception \& Psychophysics, 60, 503-510.

Sawusch, J. R. (1977). Peripheral and central processes in selective adaptation of place of articulation in stop consonants. Journal of the Acoustical Society of America, 62, 738-750.

Sawusch, J. R., \& Jusczyk, P. (1981). Adaptation and contrast in the perception of voicing. Journal of Experimental Psychology. Human Perception and Performance, 7, 408-421.

Schirmer, A., \& Kotz, S. A. (2003). ERP evidence for a sex-specific Stroop effect in emotional speech. Journal of Cognitive Neuroscience, 15, $1135-1148$

Schweinberger, S. R., Casper, C., Hauthal, N., Kaufmann, J. M., Kawahara, H., Kloth, N., \& Zäske, R. (2008). Auditory adaptation in voice perception. Current Biology, 18, 684-688. doi:10.1016/ j.cub.2008.04.015

Schweinberger, S. R., Walther, C., Zäske, R., \& Kovacs, G. (2011). Neural correlates of adaptation to voice identity. British Journal of Psychology, 102, 748-764.

Simpson, A. P., \& Ericsdotter, C. (2007). Sex-specific differences in f0 and vowel space. In Proceedings of the 16th International Congress of Phonetic Sciences (pp. 933-936). Saarbrücken: Saarland University.

Smith, E. L., Grabowecky, M., \& Suzuki, S. (2007). Auditory-visual crossmodal integration in perception of face gender. Current Biology, 17, 1680-1685.

Studdert-Kennedy, M. (1980). Speech-perception. Language and Speech, 23, 45-65.

Sussman, J. E. (1993). Focused attention during selective adaptation along a place of articulation continuum. Journal of the Acoustical Society of America, 93, 488-498.

Suzuki, S., \& Grabowecky, M. (2003). Attention during adaptation weakens negative afterimages. Journal of Experimental Psychology. Human Perception and Performance, 29, 793807.

Tartter, V. C., \& Eimas, P. D. (1975). Role of auditory feature detectors in perception of speech. Perception \& Psychophysics, 18, 293-298.

Titova, N., \& Näätänen, R. (2001). Preattentive voice discrimination by the human brain as indexed by the mismatch negativity. Neuroscience Letters, 308, 63-65.

Treisman, A. M. (1964). Verbal cues, language, and meaning in selective attention. The American Journal of Psychology, 77, 206-219.

von Kriegstein, K., Eger, E., Kleinschmidt, A., \& Giraud, A. L. (2003). Modulation of neural responses to speech by directing attention to voices or verbal content. Cognitive Brain Research, 17, 48-55.

Webster, M. A., Kaping, D., Mizokami, Y., \& Duhamel, P. (2004). Adaptation to natural facial categories. Nature, 428, 557-561.

Zäske, R., \& Schweinberger, S. R. (2011). You are only as old as you sound: Auditory aftereffects in vocal age perception. Hearing Research, 282, 283-288. doi:10.1016/j.heares.2011.06.008

Zäske, R., Schweinberger, S. R., Kaufmann, J. M., \& Kawahara, H. (2009). In the ear of the beholder: Neural correlates of adaptation to voice gender. European Journal of Neuroscience, 30, 527-534.

Zäske, R., Schweinberger, S. R., \& Kawahara, H. (2010). Voice aftereffects of adaptation to speaker identity. Hearing Research, $268,38-45$. 